\title{
Das Kunsthistorische Museum Wien als Schmerzmuseum
}

\author{
Heber Ferraz-Leite
}

Eingegangen: 24. Januar 2018 / Angenommen: 24. Januar 2018 / Online publiziert: 14. März 2018

(c) Der/die Autor(en) 2018. Dieser Artikel ist eine Open-Access-Publikation.

Zusammenfassung Die Gemäldegalerie des Kunsthistorischen Museums Wien besitzt repräsentative Werke der Historienmalerei (Darstellungen geschichtlicher Ereignisse, Religion und mythologischen Themen). Nicht die technischen oder malerischen Eigenschaften dieser Werke, sondern eine besondere Thematik wird hier analysiert. In einer Serie von $529 \mathrm{im}$ Mai 2017 ausgestellten Bildern waren 85 Abbildungen von Schmerzleidenden. Auftraggebende waren die politisch und religiös Mächtigen. Die Bilder sollten sich an ihren Geschmack und ihre Ziele anpassen. Hier wird versucht, die Ursachen und Gründe für die Darstellungen des Leidens zu erklären. Die häufigsten waren die Verherrlichung eines Herrschers sowie die strategische Unterstützung der Sozialisation und Erziehung durch Verinnerlichung der religiösen Werte, um die Macht zu sichern. Der Schmerz wird als Weg zur Seligkeit, aber auch als berechtigte Bestrafung abgebildet. Die Neurophysiologie der kognitiven Reaktionen gegenüber Schmerzdarstellungen wird noch erforscht.

Schlüsselwörter Schmerz · Kunst · Bilder · Religion · Kunsthistorisches Museum Wien

\section{The Kunsthistorisches Museum Wien as a museum of pain}

Summary The picture gallery of the Kunsthistorisches Museum Wien (Vienna Museum of Art History) has works representative of historical painting (depictions of historical events, religion, and mythological themes). Not the technical or pictorial qualities of these works, but a special subject matter is analyzed

Ass. Prof. Dr. H. Ferraz-Leite, MSc. ( $ه)$

Gentzgasse 10/5/16, 1180 Wien, Österreich

Heber.Ferraz@meduniwien.ac.at herein. In a series of 529 pieces of art exhibited in May 2017, 85 show illustrations of human suffering. The art commissioners were the political and religious leaders. The images had to be adapted to their taste and goals. We looked for reasons to explain the depiction of suffering. The most frequents reasons were glorification of a ruler, as well as strategic support of socialization and education by internalizing religious values, in order to secure power. Pain is portrayed as a way to salvation, but also as a just punishment. The neurophysiology of cognitive responses to pain representations are still under investigation.

Keywords Pain · Art · Pictures · Religion · Kunsthistorisches Museum Vienna

\section{Einführung}

Die Kunst hat sich immer mit der Darstellung menschlicher Erlebnisse beschäftigt. Der Schmerz ist eine komplexe subjektive Sinnes- und Gefühlserfahrung, die das Leben intensiv prägt und als ein biopsychosoziales Gesamtphänomen zu verstehen ist [1]. Die Gründe für die künstlerischen Darstellungen des Schmerzes sind sehr unterschiedlich und reflektieren die Lebensart, das Gedankengut, die religiösen Einstellungen und die Kultur der Zeit.

Die außergewöhnliche Gemäldegalerie des Kunsthistorischen Museums Wien (KHM Wien) hat ihren Ursprung in der Sammlung des Hauses Habsburg. Die Schwerpunkte der Galerie sind die venezianische Malerei des 16. Jahrhunderts (Tizian, Veronese, Tintoretto), die flämische Malerei des 17. Jahrhunderts (Peter Paul Rubens, Anthonis van Dyck) sowie die altniederländische (Jan van Eyck, Rogier van der Weyden) und die altdeutsche Malerei (Albrecht Dürer, Lucas Cranach). 


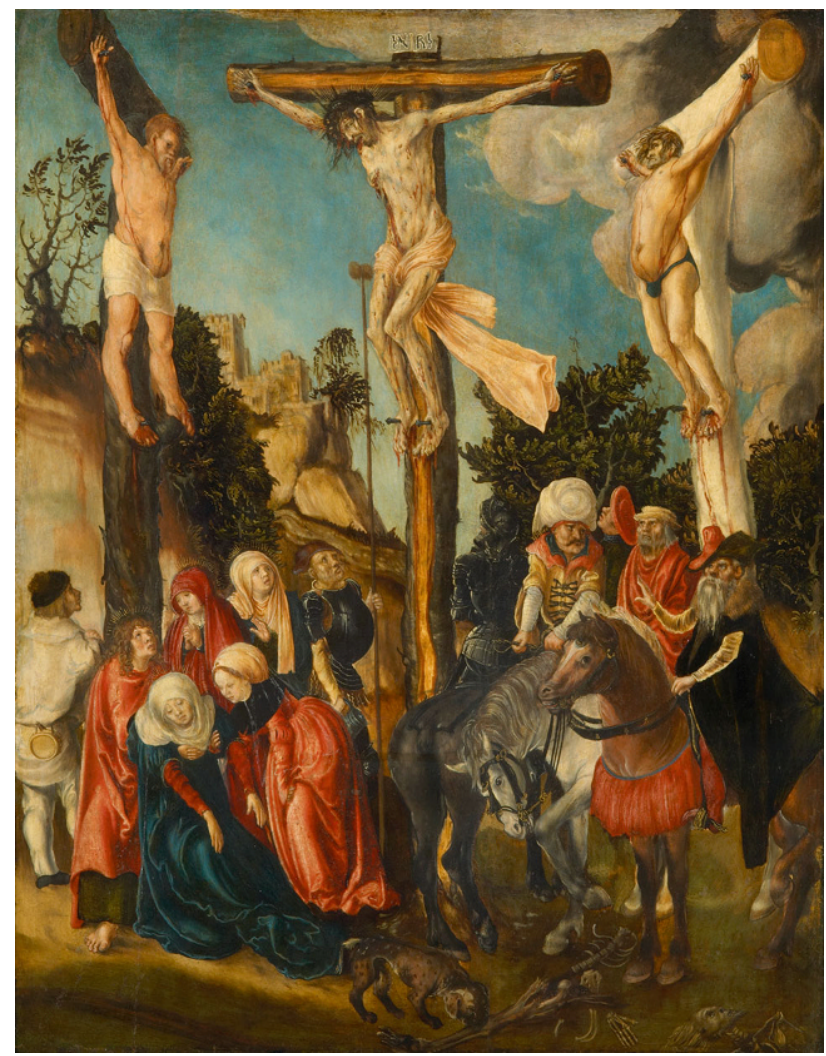

Abb. 1 „Kreuzigung Christi“ von Lucas Cranach d. Ä. (1500/01). (C) Kunsthistorisches Museum Wien. Abdruck mit freundl. Genehmigung

Ein weiterer herausragender Aspekt ist die Ausstellung von Werken von Pieter Bruegel d. Ä., und Meisterwerke von Vermeer, Rembrandt, Raffael, Caravaggio, Velázquez und der italienischen Barockmalerei [2].

Eine große Anzahl der Bilder stellen die Schmerzen der Menschen dar sowie ihre Ursachen und Konsequenzen [3]. Man beschäftigt sich nicht nur mit der Art der Leiden, sondern auch welchen Sinn bzw. welche Ziele diese damaligen künstlerischen Darstellungen verfolgten.

\section{Material und Methoden}

Es wurden 529 Werke, gezeigt im Mai 2017 in zufällig ausgewählten 14 Sälen und 14 Kabinetten des ersten Stocks des KHM Wien, gründlich beschrieben. Fotos und Informationen der ausgestellten Bilder sind auf der Homepage des Museums publiziert. Zusätzlich ausgewählte Details der Bilder wurden fotografisch von mir dokumentiert. Mehrere Museumsführer und Bücher über die alten Meister wurden herangezogen. Ein Protokoll nach genaueren Definitionen von körperlichen und seelischen Aggressionen, akuter, chronischer, und seelischer Schmerzen einerseits sowie religiöser, mythologischer, volkstümlicher und historischer Thematik andererseits wurde ausgefüllt und später ausgewertet.

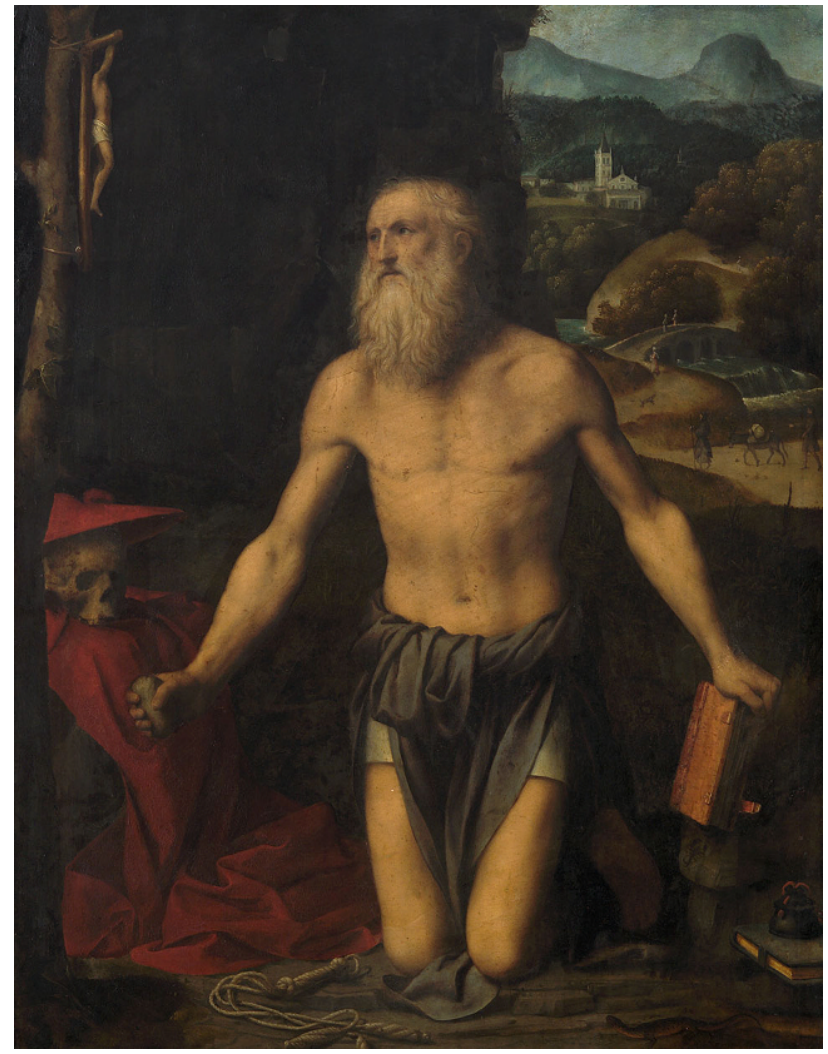

Abb. 2 „HI. Hieronymus“ von Bernardino Luini (nach 1525). (c) Kunsthistorisches Museum Wien. Abdruck mit freundl. Genehmigung

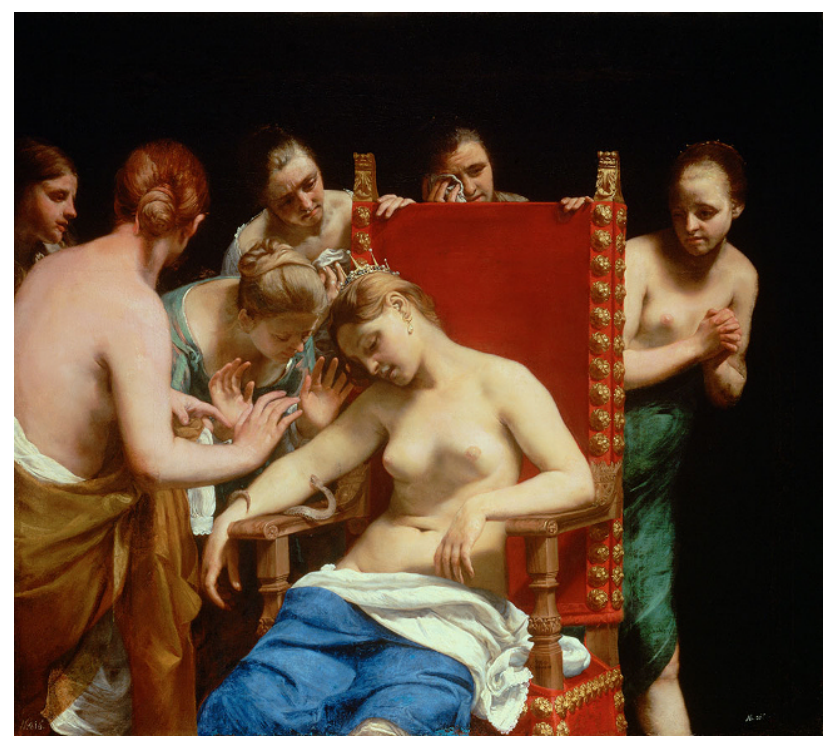

Abb. 3 „Selbstmord der Kleopatra“ von Guido Cagnacci (1661-1662). () Kunsthistorisches Museum Wien. Abdruck mit freundl. Genehmigung 
Abb. 4 „Die Gehbehinderte in ,Kampf zwischen Karneval und Fasten" "von Pieter Bruegel d. Ä. (1559).

(C) Kunsthistorisches Museum Wien. Abdruck mit freundl. Genehmigung

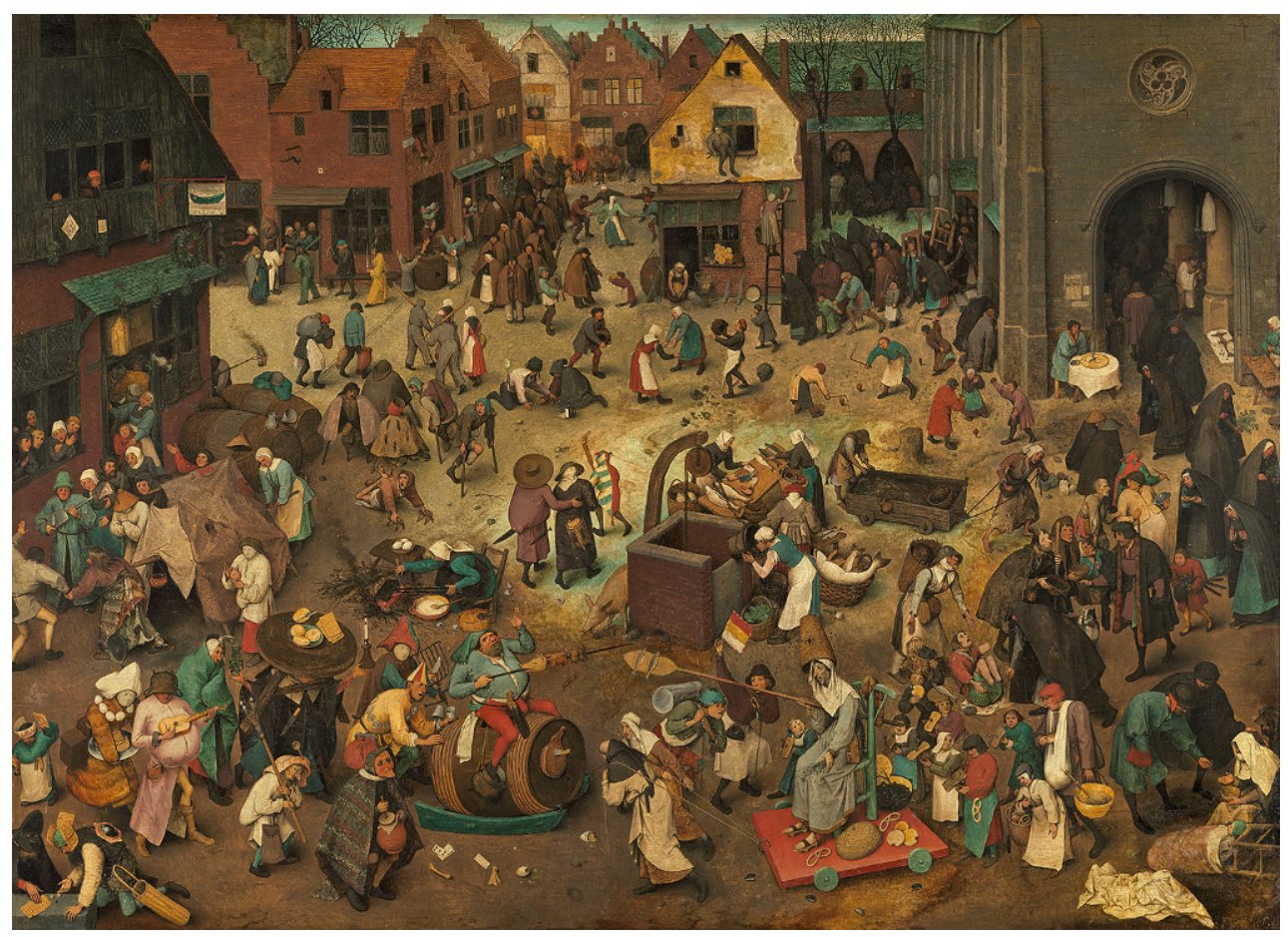

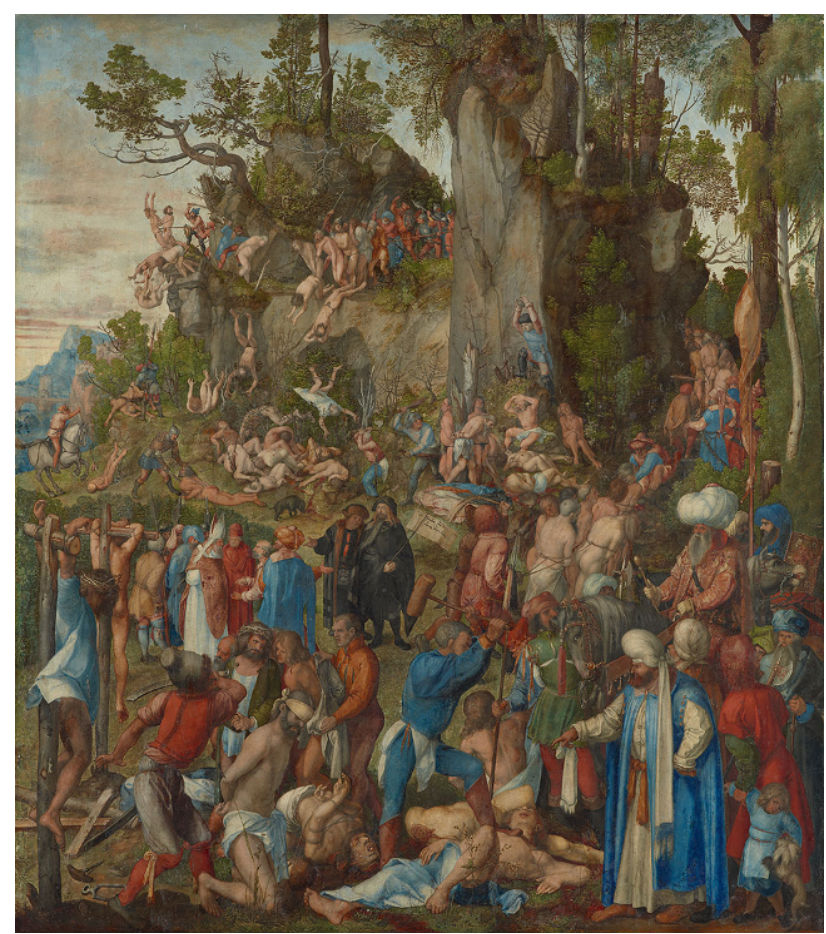

Abb. 5 „Marter der zehntausend Christen“ von Albrecht Dürer (1508). () Kunsthistorisches Museum Wien. Abdruck mit freundl. Genehmigung



Abb. 6 „Bethlehemitischer Kindermord“ von Alessandro Turchi, gen. L'Orbetto (1610/1615). () Kunsthistorisches Museum Wien. Abdruck mit freundl. Genehmigung 
Abb. 7 „Jüngstes Gericht“ von Frans de Vriendt, gen. Floris (1565). (c) Kunsthistorisches Museum Wien. Abdruck mit freundl. Genehmigung

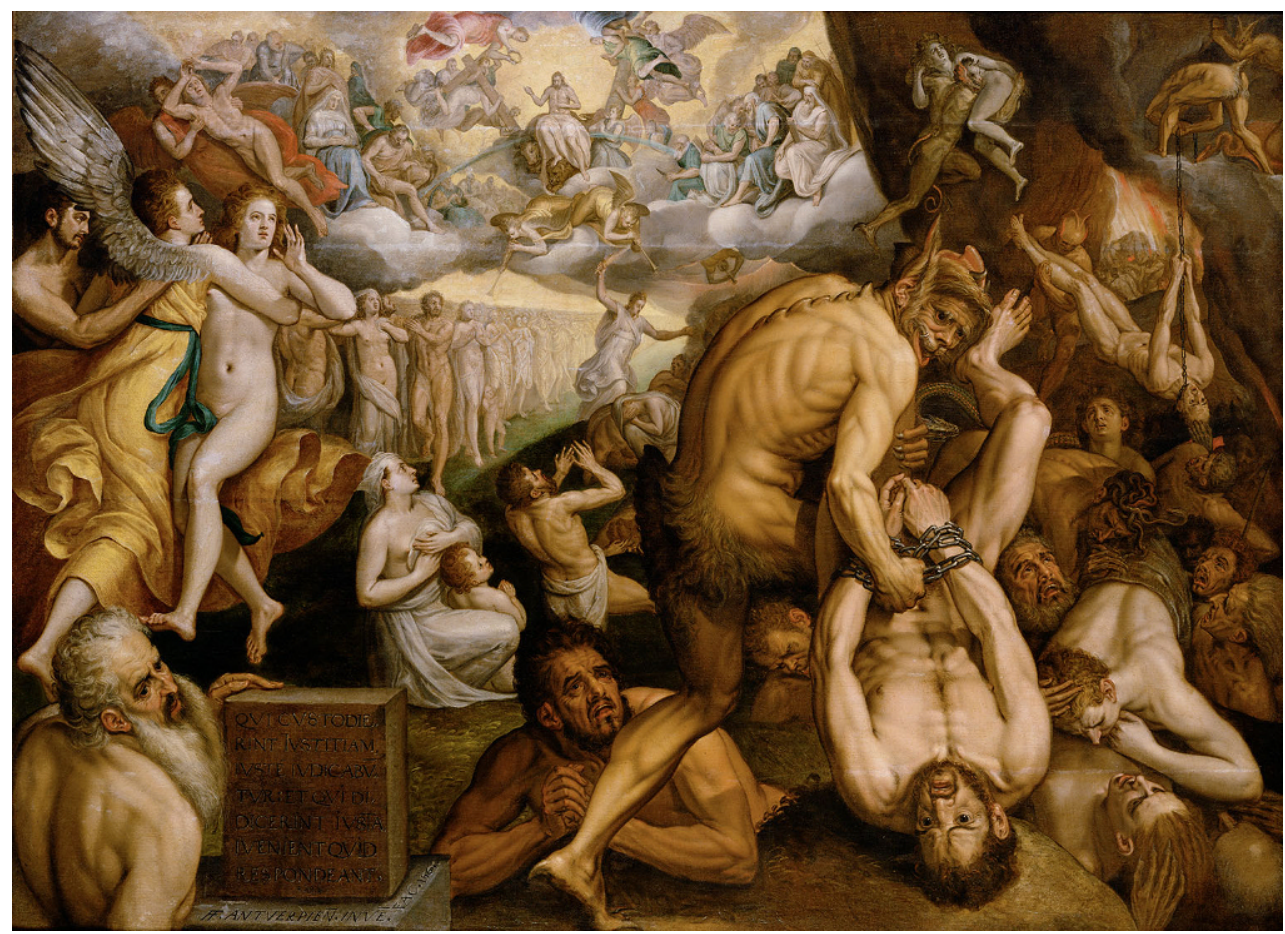

\section{Ergebnis}

Darstellungen von Schmerzleidenden oder Gestorbenen nach einem qualvollen Tod wurden in 85 Bildern (16\%) gefunden.

Körperliche Aggression bzw. Verletzungen als Ursache akuter Schmerzen werden in 58 Bildern (11\%) dargestellt (Abb. 1), bei zehn dieser Bilder handelt es sich um eine Autoaggression (Abb. 2); Ein Selbstmord wird in drei der Bilder gezeigt (Abb. 3). Sechs Bilder zeigen eine klare Erkrankung, häufig des Bewegungsapparats, als Grund für vermutlich chronische Schmerzen (Abb. 4). Demütigungen, Hilflosigkeit und soziale Ausgrenzung sind in 32 Bildern dargestellt, häufig auch zusammen mit körperlichen Aggressionen (Abb. 5).

Diese Aggressionen geschehen:

a) im Laufe der Ausübung brutaler sozialer, bzw. politischer Macht (Abb. 6);

b) als Strafe Gottes (Abb. 7), wobei nicht nur Menschen bestraft werden, sondern auch ungehorsame Engel (Abb. 8);

c) während kriegerischer Auseinandersetzungen (Abb. 9);

d) durch Gewalt der Natur (Abb. 10);

e) Unfälle (Abb. 10);

f) Erkrankungen (Abb. 11).
Die seelischen Schmerzen, hauptsächlich Reue, sind Inhalt von 21 Bildern, wobei Maria Magdalena die am meisten dargestellte bereuende Sünderin ist (Abb. 12).

Schmerzleidende kommen in 67 Werken mit katholischer bzw. jüdischer religiöser Thematik vor sowie auch in 7 mythologischen Darbietungen und in 6 Bildern mit einem geschichtlichen Inhalt. Vier Bilder mit philosophischen Grundgedanken oder Lebenswahrnehmungen reflektieren auch über menschlichen Schmerz (Abb. 13).

Die grausamen Folterungen und Tötungen sind ein besonderes Kapitel. So wird in dem ursprünglich bestellten Altarbild für eine Seitenkapelle des Doms von Salzburg der Märtyrertod des Seligen Thiemo dargestellt, indem ihm die Gedärme mit einer Darmspindel aus dem Leib gezogen werden (Abb. 14).

Mehrere Bilder repräsentieren Enthauptungen, die von Helden, bzw. Heldinnen verübt worden sind. Die häufigsten Darstellungen sind von David mit dem Kopf von Goliath und Judith mit dem Kopf von Holofernes. Aber auch die böse hübsche Salome wird mit dem Kopf von Johannes dem Täufer befriedigt (Abb. 15, 16 und 17).

Ein tröstendes Bild zeigt David, der vor dem leidenden König Saul ein Saiteninstrument spielt (Abb. 18). 


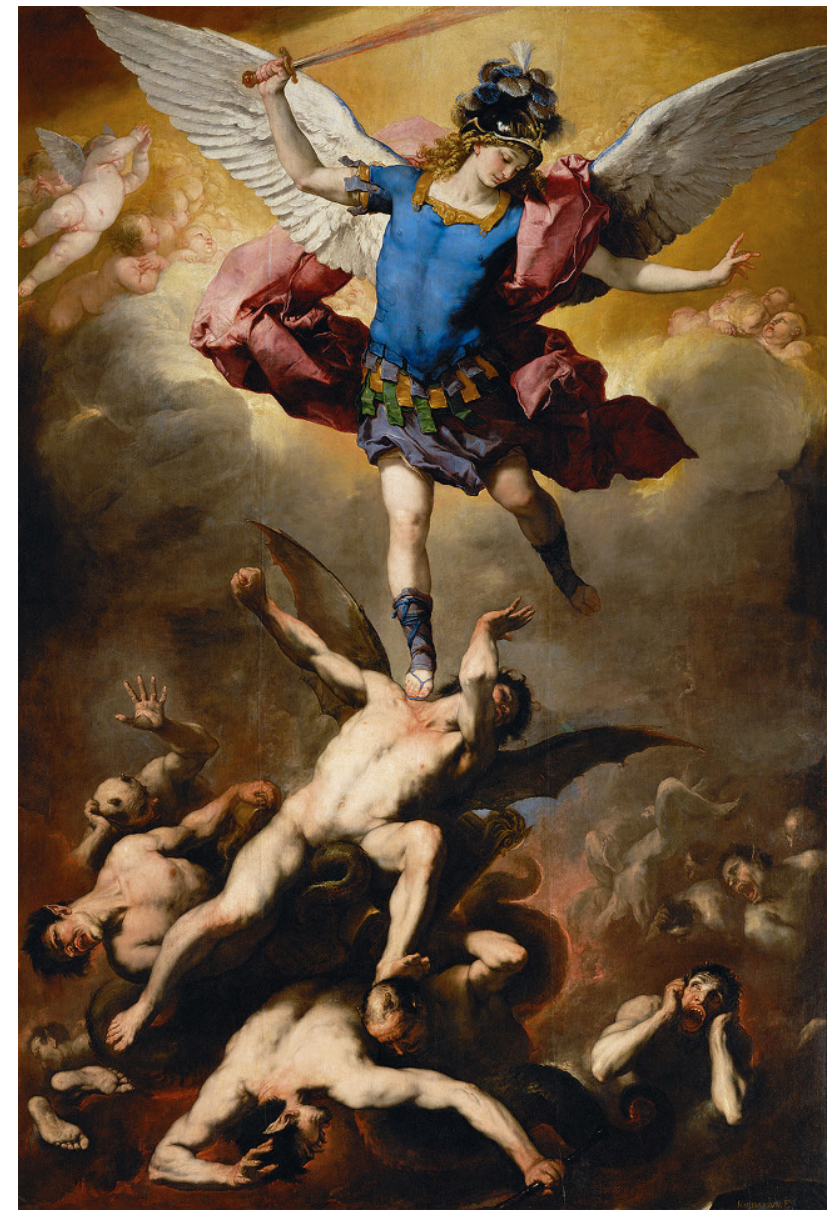

Abb. 8 „Der Erzengel Michael stürzt die Abtrünnigen Engel in den Abgrund" von Luca Giordano (1664). (C) Kunsthistorisches Museum Wien. Abdruck mit freundl. Genehmigung

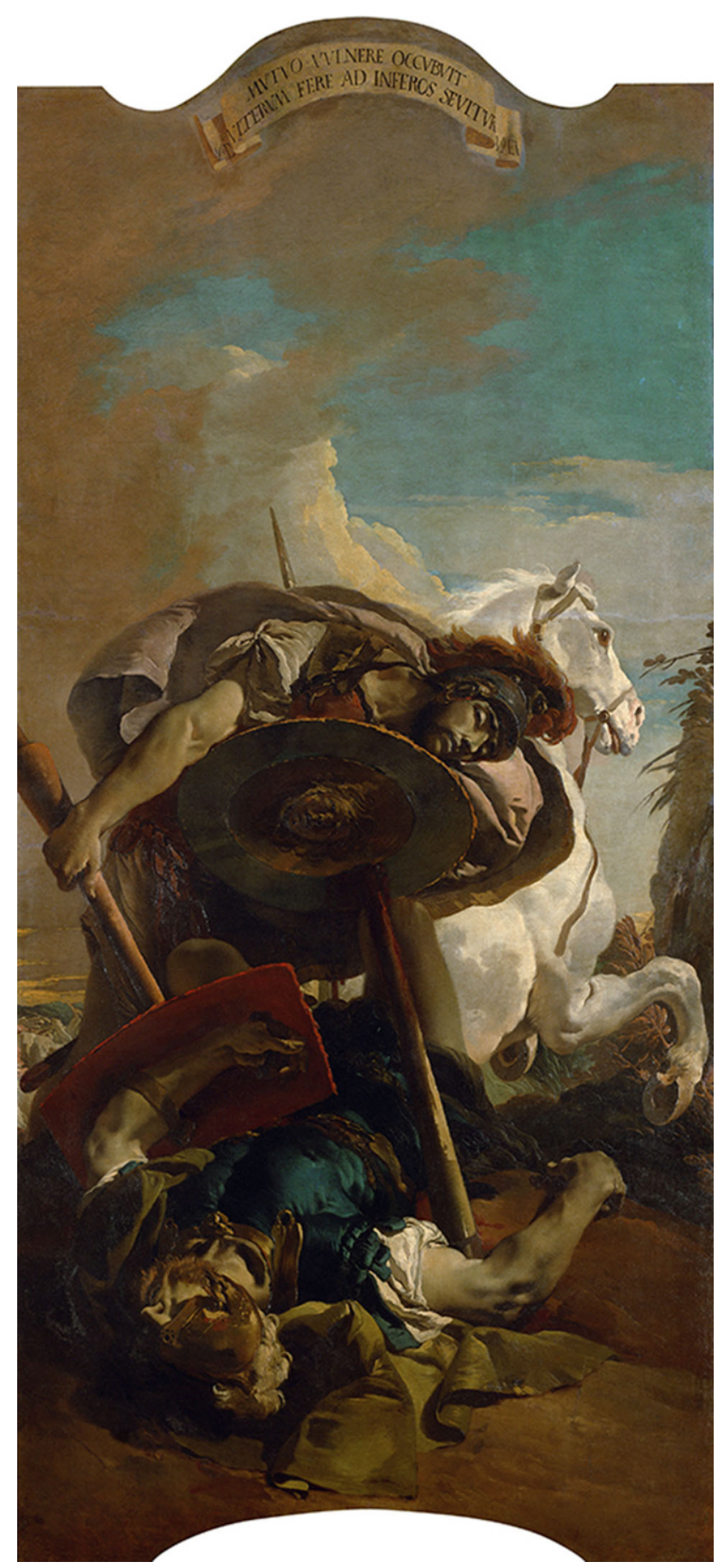

Abb. 9 „Tod des Konsuls L. J. Brutus im Zweikampf mit Aruns" von Giambattista Tiepolo (1728/1730). (C) Kunsthistorisches Museum Wien. Abdruck mit freundl. Genehmigung 


\section{themenschwerpunkt}

Abb. 10 „Wunderrettung durch einen Dominikanerheiligen" von Francesco Guardi (1763). (C) Kunsthistorisches Museum Wien. Abdruck mit freundl. Genehmigung

Abb. 11 „Die Pest in Venedig" von Antonio Zanchi (1666). (c) Kunsthistorisches Museum Wien. Abdruck mit freundl. Genehmigung
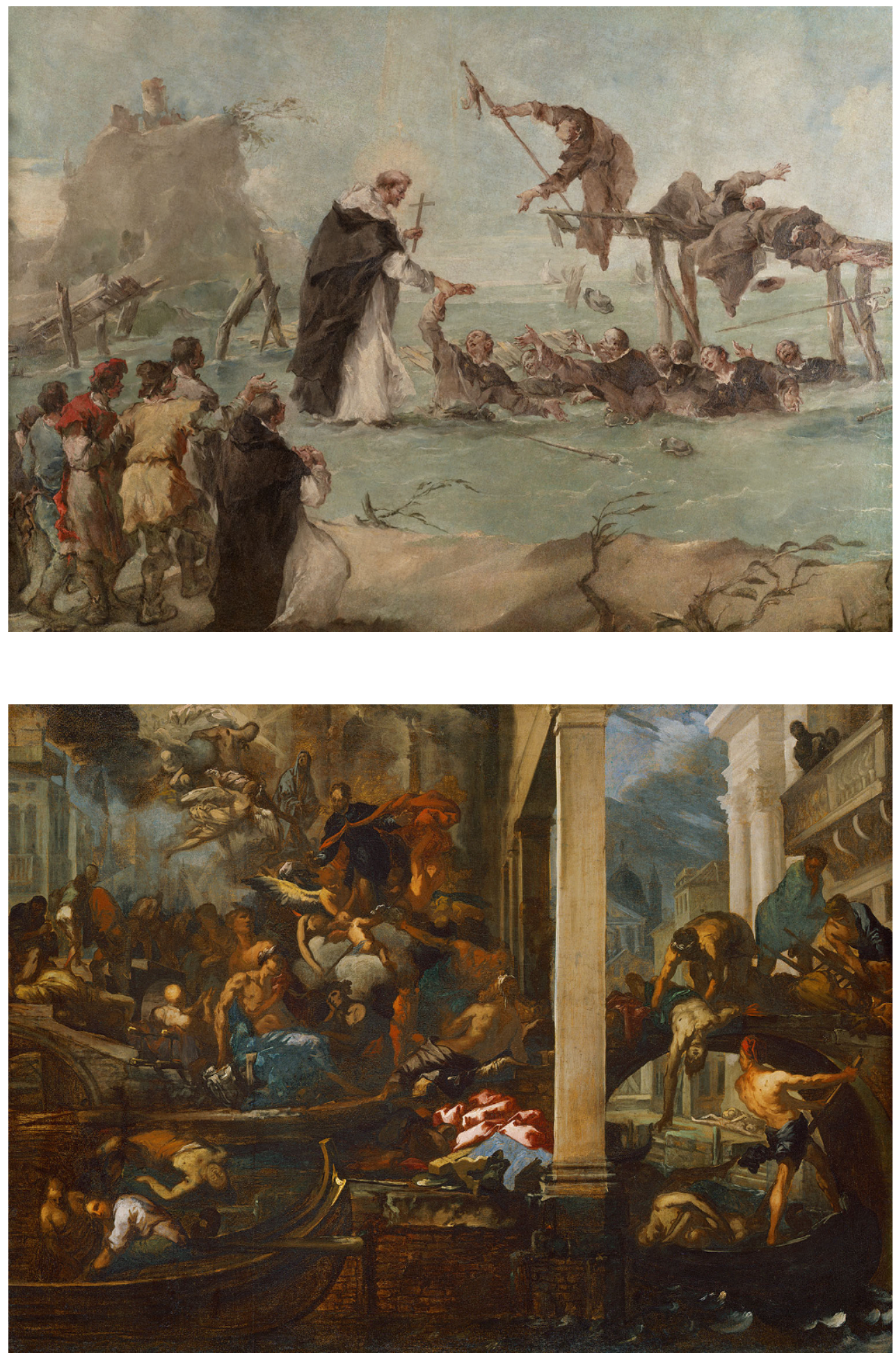


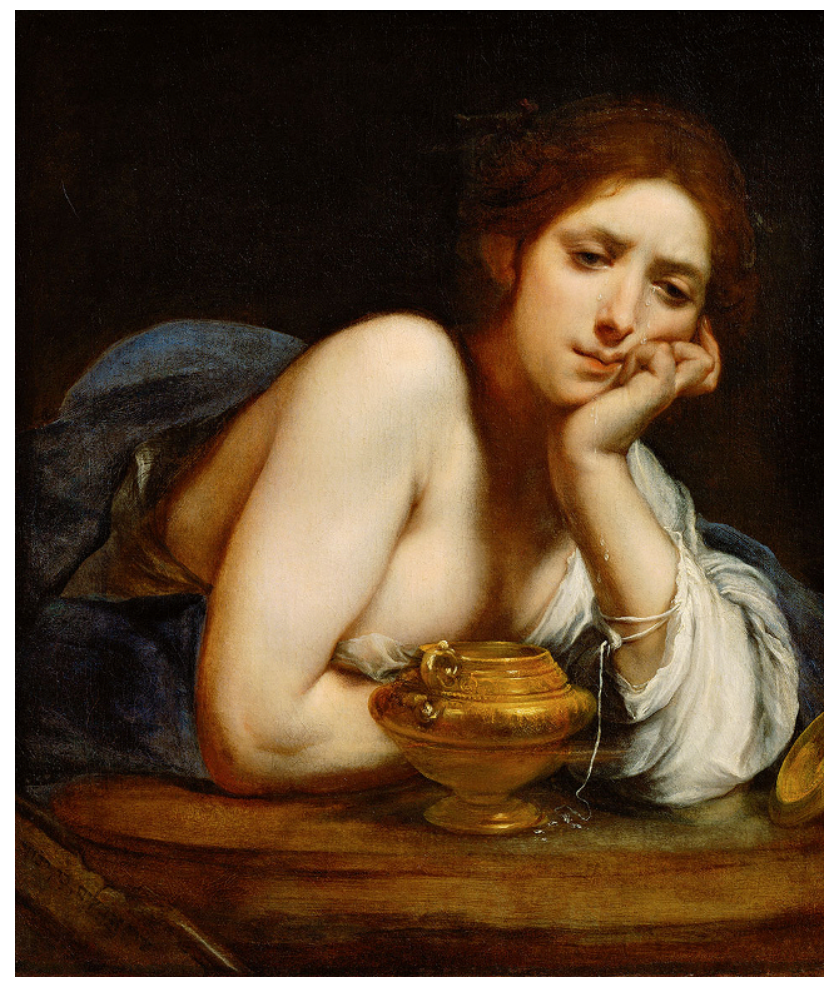

Abb. 12 „HI. Maria Magdalena“ von Francesco Furini (1630/35). (C) Kunsthistorisches Museum Wien. Abdruck mit freundl. Genehmigung

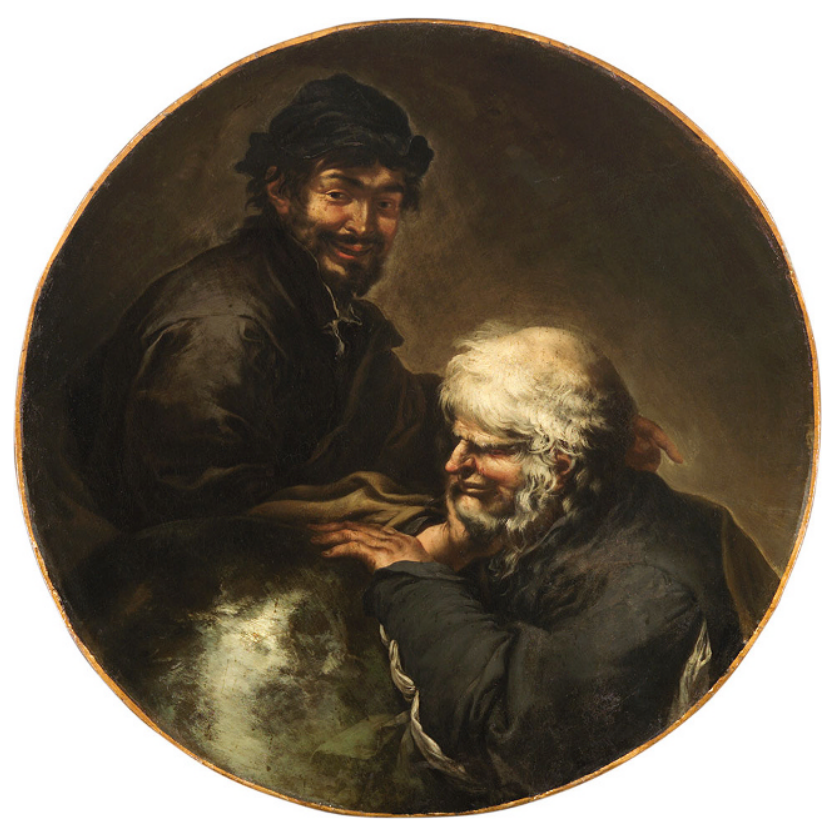

Abb. 13 „Heraklit und Demokrit“ von Salvator Rosa (1645/49). (C) Kunsthistorisches Museum Wien. Abdruck mit freundl. Genehmigung

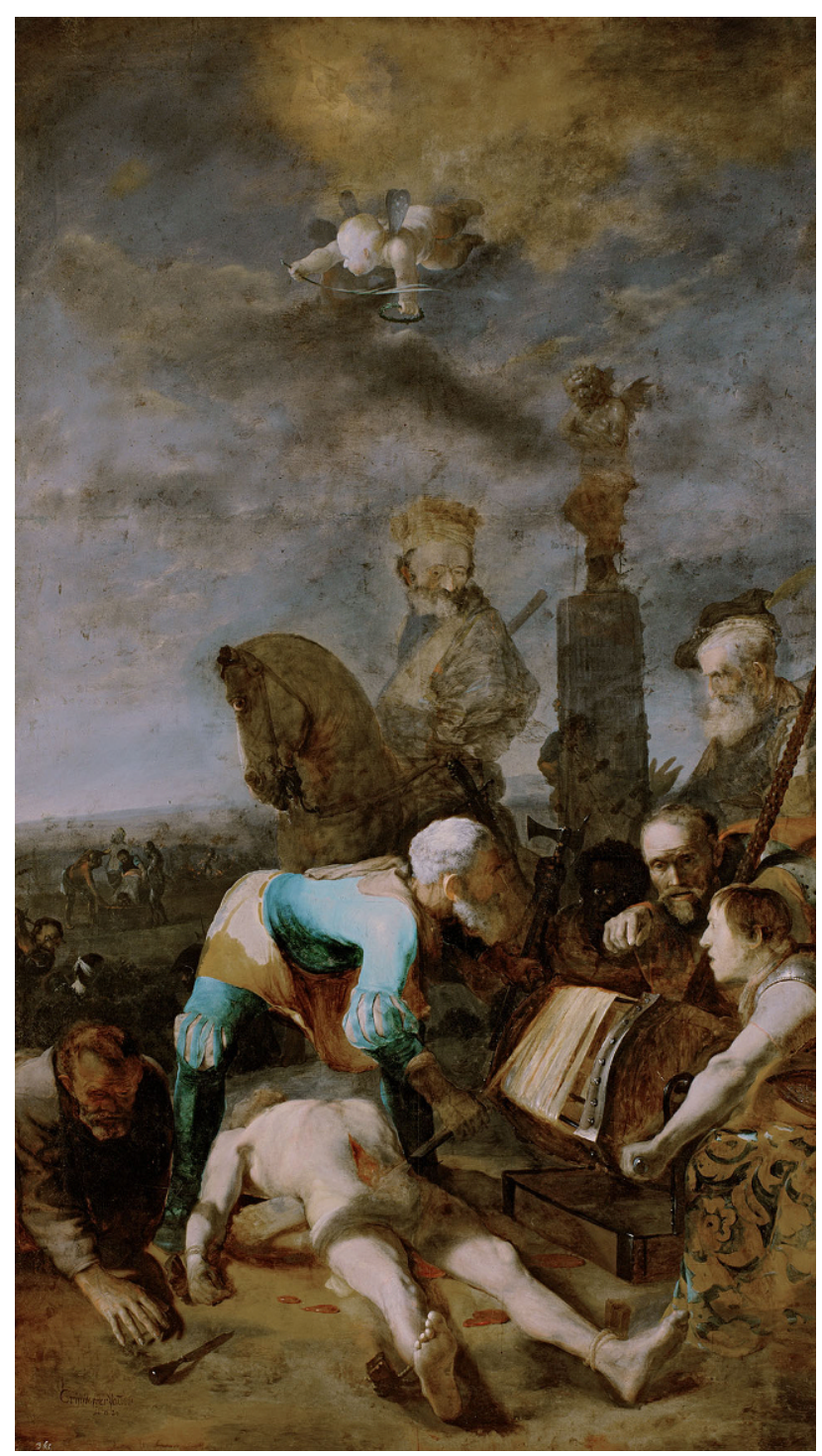

Abb. 14 „Marter des HI. Thiemo" von Christoph Paudiß (1662). (C) Kunsthistorisches Museum Wien. Abdruck mit freundl. Genehmigung

\section{Diskussion}

Diese außergewöhnliche Sammlung konfrontiert uns hauptsächlich mit der Lebensart, dem Gedankengut, den religiösen Einstellungen und der Kultur der damaligen mitteleuropäischen Zeit. Die Auftraggeber waren meist wohlhabende Prälaten, Fürsten, Kaufleute oder Personengruppen, wie Ordensgemeinschaften und Bruderschaften. Inhalt und Zweck der Kunst sollten ihren Interessen dienen bzw. deren Geschmack treffen [4]. Man könnte sich fragen, welche Rolle diese häufigen Darstellungen der Schmerzleidenden verfolgten. Sie erwecken Angst, Mitleid und Andacht. Man kann postulieren, dass solche Bilder dem Prozess der Sozialisation und Erziehung dienten. Belehrung und Warnung durch Bilder tragen zu Verinnerlichung (Aneignung) der Werte, Sitten, Normen und sozia- 


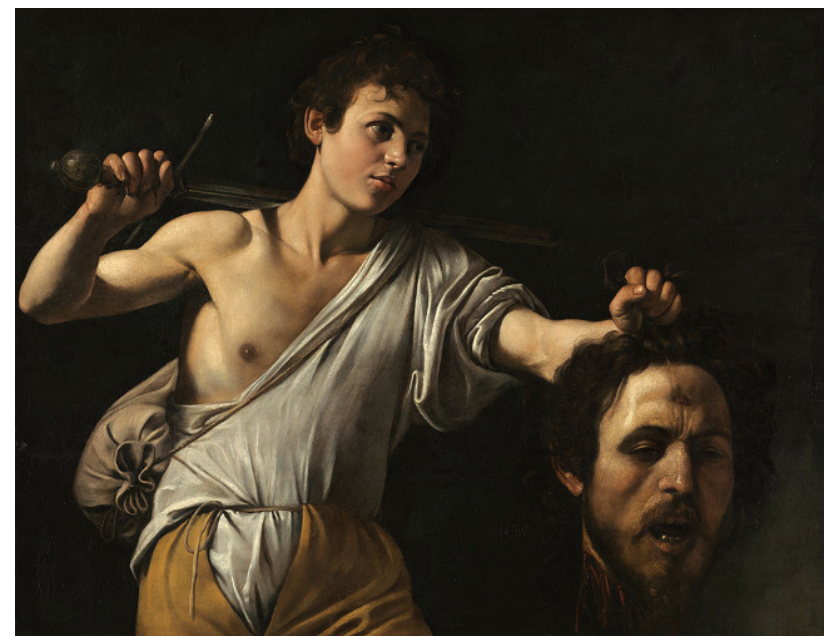

Abb. 15 „David mit dem Haupt des Goliath“ von Caravaggio (1600/01). (C) Kunsthistorisches Museum Wien. Abdruck mit freundl. Genehmigung

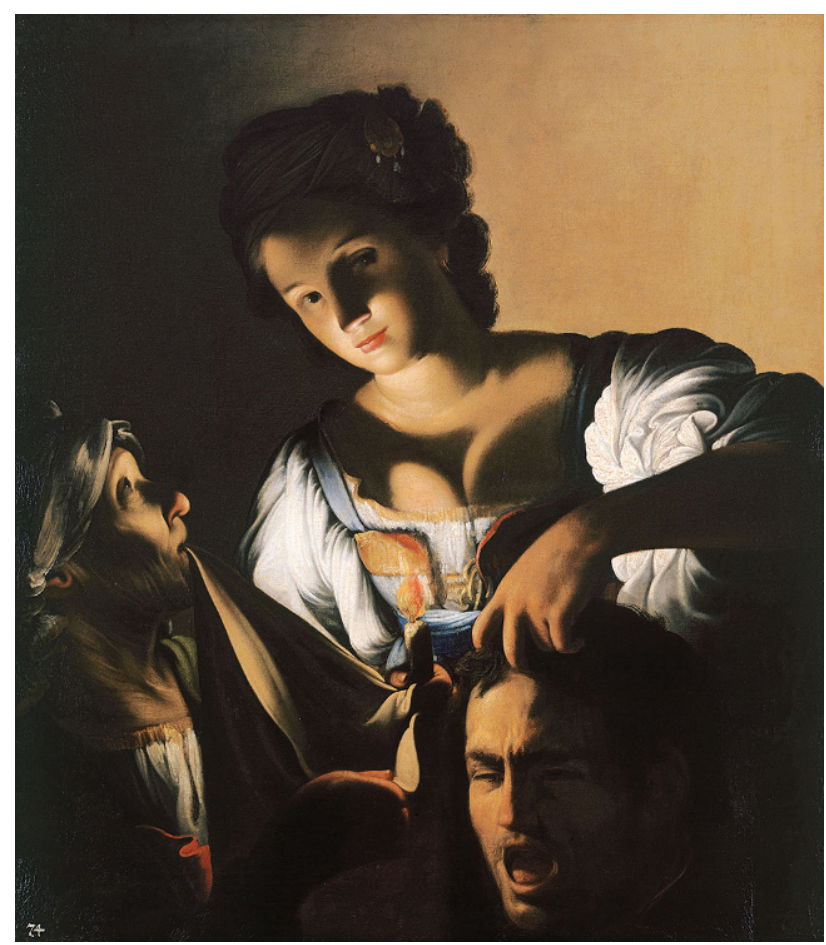

Abb. 16 "Judith mit dem Haupt des Holofernes“ von Carlo Saraceni (1610/15). (c) Kunsthistorisches Museum Wien. Abdruck mit freundl. Genehmigung

len Rollen, die zur Religion und zu den politischen Systemen gehörten, bei.

So ist Christus die Hauptfigur des Leidens: Wenn Gottes Sohn so gelitten hat, kann man es auch von mir verlangen! Ein solidarisches gemeinsames Bewusstsein, das Glaubensvorstellungen umfasst, wird gefordert. Gleichzeitig wird ein irrationales Schuldgefühl des Betrachters verstärkt.

Bilder, die Schmerzen darstellen und Empathie hervorrufen, werden in wissenschaftlichen, experimen-

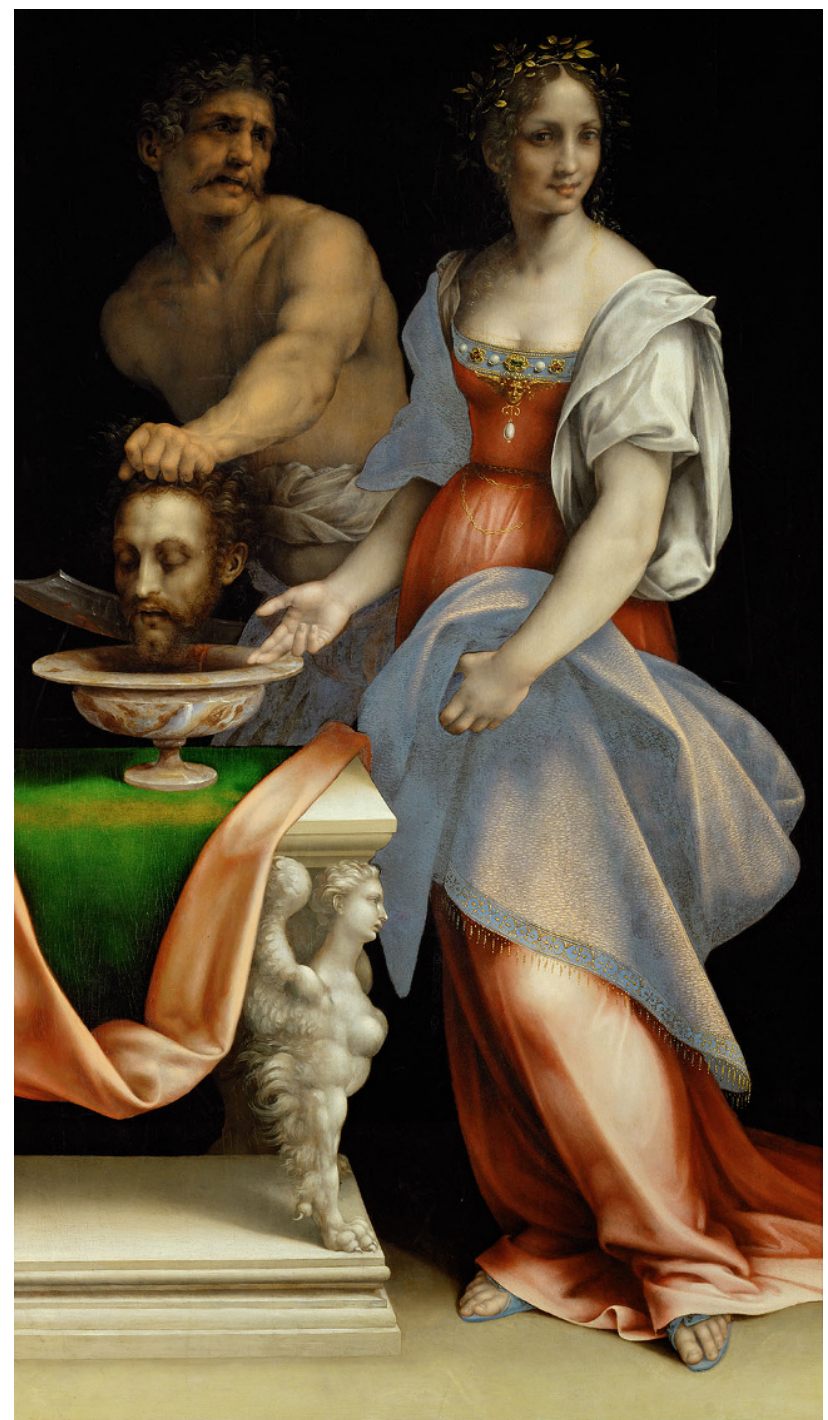

Abb. 17 „Salome mit dem Haupt Johannes des Täufers“ von Cesare da Sesto (1512/16). (C) Kunsthistorisches Museum Wien. Abdruck mit freundl. Genehmigung

tellen Studien verwendet, um die zugrunde liegenden neuronalen Prozesse zu erforschen. Man postuliert, dass eventuell die Beteiligung des Spiegelneuronensystems einbezogen wird. Das wurde aber bis jetzt $\mathrm{zu}$ wenig wissenschaftlich untermauert. Bewiesen ist aber, dass solche Bilder kognitive Reaktionen erwecken [5].

Die am meisten dargestellten Momente von Christus Leben sind sein Kreuzweg und sein Tod, wo viele körperliche und seelische Aggressionen gemeinsam vorkommen. Die seelischen Schmerzen seiner Mutter, seines irdischen Vaters, seiner Freunde und Schüler sind auch oft bei den „Kreuzabnahmen“ und der „Beweinung Christi“ dargestellt.

Einige Bilder zeigen in exemplarischer Weise die Schmerzen der Bestraften, die sich der Übertretungen von Normen oder Anzweiflung von Werten, die das System beanspruchte, schuldig gemacht haben. Es be- 
Abb. 18 „David spielt vor Saul" von Bernardo Cavallino (1645). (C) Kunsthistorisches Museum Wien. Abdruck mit freundl. Genehmigung

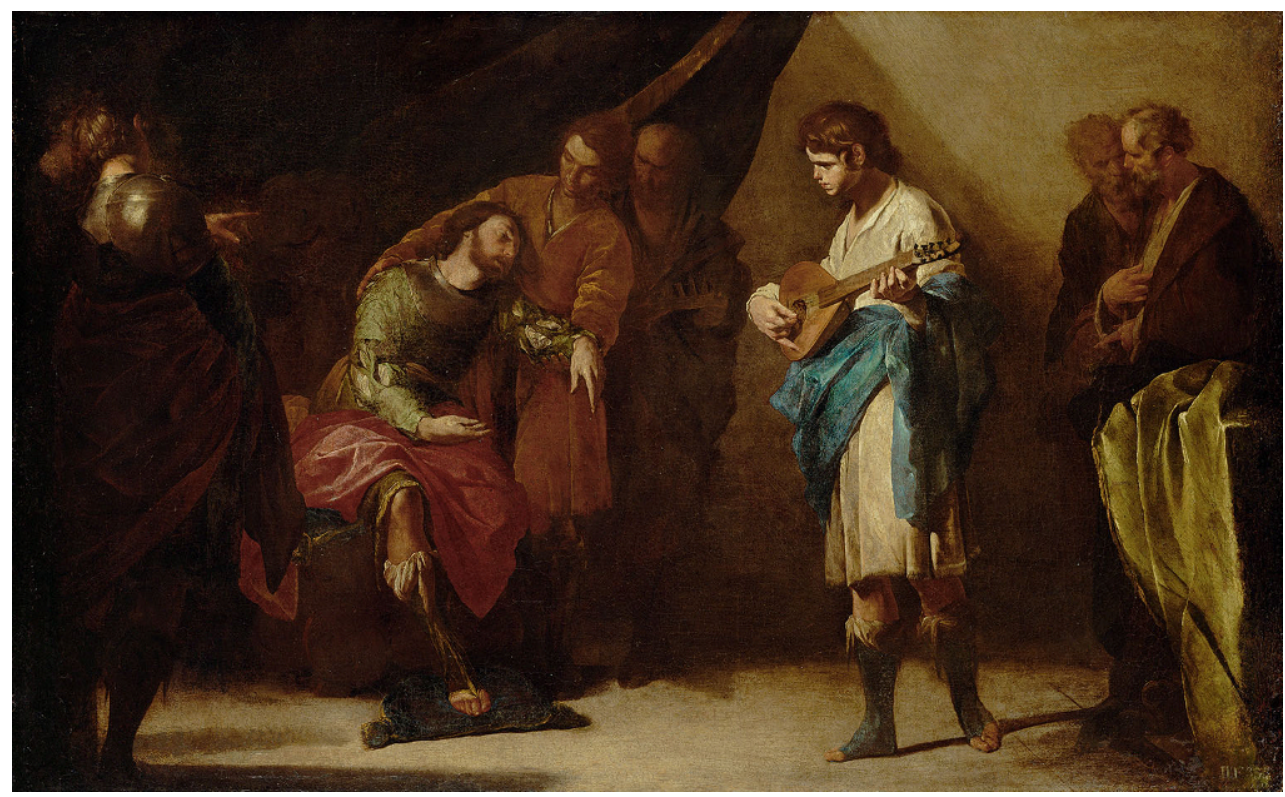

steht immer eine erhebliche Ungleichheit der Macht zwischen Bestrafenden und Bestraften.

Der seelische Schmerz der Reue kommt oft zur Darstellung (Abb. 12). Diese Bilder provozieren die Reue. Die Werte, die übertreten sind, werden internalisiert und die/der Schuldige bestraft sich selbst mit dem Schmerz der Reue. Es geht darum, den Menschen „schuldig“ zu machen. Der Mensch ist „wissend“, das ist die Urschuld, die auf Adam und Eva zurückgeht.

Die Darstellungen von Enthauptungen sind die stärkste Form des Gräuels. In psychoanalytischen Analysen stellen sie aber eine besondere Art von Grausamkeit dar, weil sich dahinter die Entmachtung verbirgt, heißt Verlust der Potenz und Ratio (Abb. 15).

Das Bild von David, der vor dem leidenden König Saul ein Instrument spielt (Abb. 17), wäre eine frühe Anerkennung der Wirkung der Musik zur Therapie der Schmerzen [6].

\section{Schlussfolgerungen}

Die Sammlung der Bildergalerie des KHM Wien ist ein beeindruckendes künstlerisches Erbe, das viele Aspekte der Gesellschaft dokumentiert. Es werden auch das Elend und der Mangel an Ressourcen zur Behandlung der Schmerzen und Krankheiten in den Jahrhunderten, aus denen diese Werke kommen, dargestellt. Der Schmerz war ein bedrohliches und teilweise unvermeidliches Vorkommnis. Anhand der religiösen Überzeugungen war der selbsterfahrene Schmerz ein Weg, um die Verzeihung der Sünde zu verdienen und eine bessere ewige Zukunft zu bekommen, ja sogar manchmal Heldentum zu erreichen. Ihre künstlerische Darstellung sollte als Warnung, Belehrung und Forderung der Internalisierung von Werten dienen, die zur herrschenden sozialen Ordnung führten. Kultur und Kunst waren (und sind noch) ein Merkmal und Teil der Herr- schaftsausübung. Sie dienten und dienen den herrschenden Klassen und der Kirche als Instrumentarien der Macht und deren Demonstration.

Da damals die Menschen (die meisten konnten nicht lesen und schreiben) sehr von optischen Eindrücken abhängig waren, muss man sich auch die starke Wirkung der Architektur der riesigen Kathedralen und der Schlösser vor Augen halten.

Erst mit der Aufklärung und Infragestellung der $\mathrm{Au}-$ toritäten und der auftretenden Säkularisierung wird diese Machtdemonstration infrage gestellt.

Hegels Behauptung „Die Kunst ist tot“ demonstriert diese dramatische Wandlung der Kunst und orientiert sich an den neuen Auftraggebern. Kunst impliziert danach eine Ausdruckform für die neue bürgerliche Käuferschicht und richtet sich an den neuen soziopolitischen und ökonomischen Systemen aus, um menschliche Bedürfnisse zu erfüllen.

Funding Open access funding provided by Medical University of Vienna.

Interessenkonflikt H. Ferraz-Leite gibt an, dass kein Interessenkonflikt besteht.

Open Access Dieser Artikel wird unter der Creative Commons Namensnennung 4.0 International Lizenz (http:// creativecommons.org/licenses/by/4.0/deed.de) veröffentlicht, welche die Nutzung, Vervielfältigung, Bearbeitung, Verbreitung und Wiedergabe in jeglichem Medium und Format erlaubt, sofern Sie den/die ursprünglichen Autor(en) und die Quelle ordnungsgemäß nennen, einen Link zur Creative Commons Lizenz beifügen und angeben, ob Änderungen vorgenommen wurden. 


\section{themenschwerpunkt}

\section{Literatur}

1. Merskey H, Bogduk N, Hrsg. IASP task force on taxonomy. Part III: pain terms, A current list with definitions and notes on usage:209-214. Seattle: IASP Press; 1994.

2. Prestel Museum Führer. Das Kunsthistorische Museum in Wien. 2007.

3. Ferraz-Leite H. Schmerz und Leiden in der Gemäldegalerie des Kunsthistorischen Museums Wiens. Schmerz Nachr. 2017;3/2017:48-9.
4. Weppelmann S. Im Spiegel der Meisterwerke. In: Cäcilia Bischoff: Meisterwerke der Gemäldegalerie.2017.S.9-23.

5. Schott GD. Pictures of pain: their contribution to the neuroscience of empathy. Brain. 2015;138(Pt3):812-20.

6. Chai PR, Carreiro S, Ranney ML, Karanam K, Ahtisaari M, Edwards R, Schreiber KL, Ben-Ghaly L, Erickson TB, Boyer EW. Music as an adjunct to opioid-based analgesia. J Med Toxicol. 2017;13(3):249. https://doi.org/10.1007/ s13181-017-0621-9. 